\title{
Synthesis, properties and potential applications of two-dimensional transition metal dichalcogenides
}

\author{
Sang A Han ${ }^{1}$, Ravi Bhatia ${ }^{2}$ and Sang-Woo Kim ${ }^{1,2^{*}}$
}

\begin{abstract}
In recent years, 2-dimensional (2D) materials such as graphene and h-BN have been spotlighted, because of their unique properties and high potential applicability. Among these 2D materials, transition metal dichalcogenides (TMDs) have attracted a lot of attention due to their unusual electrical, optical, and mechanical properties. Also, TMDs have virtually unlimited potential in various fields, including electronic, optoelectronic, sensing, and energy storage applications. For these various applications, there are many methods for sample preparation, such as the mechanical, liquid exfoliation and chemical vapor deposition techniques. In this review, we introduce the properties, preparation methods and various applications of TMDs materials.
\end{abstract}

Keywords: Two-dimensional materials; Transition metal dichalcogenides; Synthesis; Electronic device; Optoelectronic device; Gas sensing device; Energy storage device

\section{Introduction}

In the last few years, a great deal of attention has been dedicated to layered two-dimensional (2D) materials, such as graphene and hexagonal boron nitride (h-BN), owing to their potential applications in various fields [1-5]. The great potential of graphene has stimulated a lot of interest in the exploration of other layered 2D nanomaterials, which can complement the requirements associated with graphene. It is well known that graphene generally exhibits semi-metallic properties, and, therefore, semiconducting and insulating 2D layered nanomaterials having structural properties akin to graphene are needed in order to integrate them into nano-electronic devices for different applications. Recently, layered transition metal dichalcogenides (TMDs), such as $\mathrm{MoS}_{2}, \mathrm{WS}_{2}, \mathrm{MoSe}_{2}$ and $\mathrm{WSe}_{2}$, have been identified as semiconducting 2D layered materials. TMDs have received significant attention because they exhibit unique electrical [6,7], optical [8-15], and mechanical [16,17] properties. Layered 2D nanostructures with atomic scale thicknesses may exhibit peculiar and fascinating properties in contrast with those of their bulk parent compounds. Both the experimental

\footnotetext{
* Correspondence: kimsw1@skku.edu

'SKKU Advanced Institute of Nanotechnology (SAINT), Center for Human Interface Nanotechnology (HINT), Sungkyunkwan University (SKKU), Suwon 440-746, Korea

${ }^{2}$ School of Advanced Materials Science and Engineering, Sungkyunkwan University (SKKU), Suwon 440-746, Republic of Korea
}

and theoretical results have shown that 2D semiconductors have exceptional properties that can result in novel and important breakthroughs in the field of nanomaterials and nanodevices.

Because of these attractive properties, there are many potential applications of TMDs materials, such as electronic devices [6,18-25], optoelectronic devices [26-29], gas sensing [18,30-34] and energy storage devices [35-42]. In this review, we introduce the properties, preparation methods and various applications of TMDs materials.

\section{Review}

\subsection{Properties of TMDs}

\subsubsection{Composition}

TMDs materials are comprised of a combination of two elements, a transition metal (M) of groups $4 \sim 10$ of the transition metal series and a chalcogen (X) such as sulfur (S), selenium (Se), or tellurium (Te). Generally, TMDs materials containing group $4 \sim 7$ transition elements have a layered structure, while those with group $8 \sim 10$ transition metals have non-layered structures. Figure 1 shows the possible layered and non-layered structures of TMDs materials. Each layer has a thickness of $6 \sim 7 \AA$, which consists of a hexagonally packed layer of metal atoms combined by weak van der Waals forces [43]. The metal atoms provide four electrons to fill the bonding states of the TMDs such that the oxidation states of the metal and 


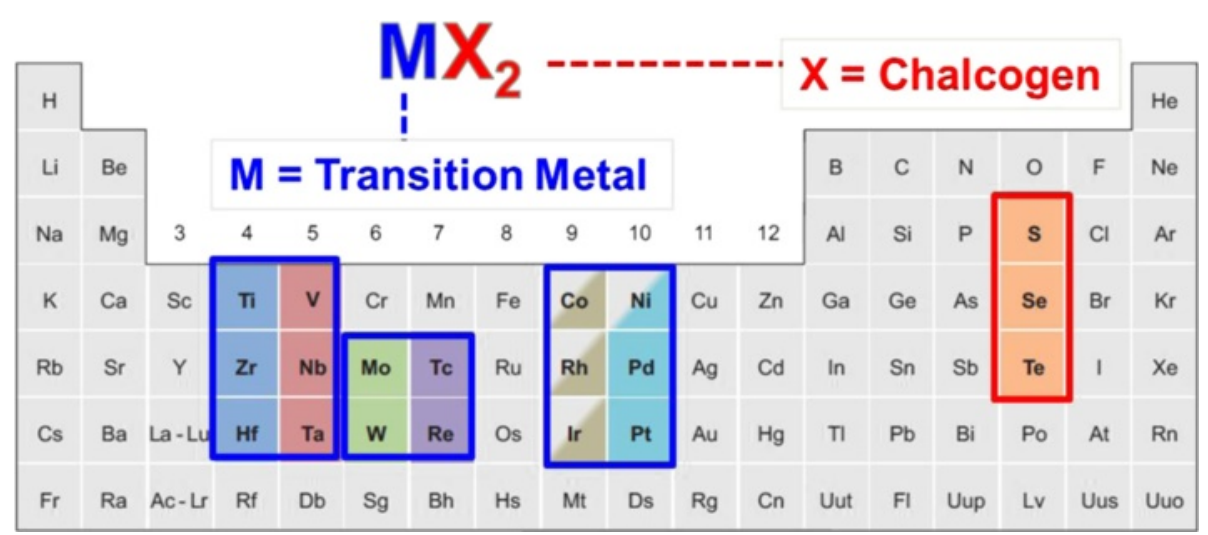

Figure 1 There are a number of possible layered structure TMDs materials consist of 16 transition metals and three chalcogen atoms. In the case of $\mathrm{Co}, \mathrm{Rh}$, Ir and $\mathrm{Ni}$, only a few layered structures are observed, for example $\mathrm{NiS}_{2}$ forms an apyrite structure, however $\mathrm{NiTe}_{2}$ forms layered structure [43].

chalcogen atoms are +4 and -2 , respectively. The bonding length of the M-X atom lies between $3.15 \AA$ and $4.03 \AA$, depending on the size of the metal and chalcogen ions.

\subsubsection{Mechanical properties}

Castellanos-Gomez et al. measured the elastic properties of a suspended portion of $\mathrm{MoS}_{2}$ nanosheets containing 5 to 25 layers [16]. The measurement of its elastic mechanical properties was performed by using the AFM tip to apply a load in the center of the suspended region of the $\mathrm{MoS}_{2}$ nanosheets (Figure $2 \mathrm{a}$ and $\mathrm{b}$ ). When the tip and sample are in contact, the elastic deformation of the nanosheets $(\delta)$, the deflection of the AFM cantilever $\left(\Delta \mathrm{z}_{\mathrm{c}}\right)$ and the displacement of the scanning piezotube of the AFM instrument $\left(\Delta \mathrm{z}_{\text {piezo }}\right)$ are related by the equation $\delta=\Delta \mathrm{Z}_{\text {piezo }}-\Delta \mathrm{Z}_{\mathrm{c}}$. The applied force is related to the cantilever deflection as $\mathrm{F}=\mathrm{k}_{\mathrm{c}} \cdot \Delta \mathrm{z}_{\mathrm{c}}$, where $\mathrm{k}_{\mathrm{c}}$ is the spring constant of the cantilever $\left(\mathrm{k}_{\mathrm{c}}=0.88 \pm 0.20 \mathrm{~N} \mathrm{~m}^{-1}\right)$. They showed that the Young's modulus of the $\mathrm{MoS}_{2}$ nanosheets is exceptionally high, i.e. $\mathrm{E}=0.33 \pm 0.07 \mathrm{TPa}$ [16]. Another experimental work also showed the mechanical properies of suspended $\mathrm{MoS}_{2}$ nanosheets. Bertolazzi et al. reported the measurement of the stiffness and breaking strength of single-layer $\mathrm{MoS}_{2}$ [17]. Exfoliated single and bilayer $\mathrm{MoS}_{2}$ nanosheets have been found to deform, and eventually become broken using AFM (Figures 2c and d). The measured in-plane stiffness of single-layer $\mathrm{MoS}_{2}$ is found to be $180 \pm 60 \mathrm{Nm}^{-1}$, and the Young's modulus of single-layer $\mathrm{MoS}_{2}$ is around $270 \pm 100 \mathrm{GPa}$ (Figure 2e). Breaking takes place at an effective strain of between 6 and $11 \%$ with an average breaking strength of $15 \pm$ $3 \mathrm{Nm}^{-1}(23 \mathrm{GPa})$. The strength of the strongest singlelayer membranes is $11 \%$ of their Young's modulus, corresponding to the upper theoretical limit, which indicates that the material is highly crystalline and almost defect-free. This results show that single-layer $\mathrm{MoS}_{2}$ could be suitable for a variety of applications, such as reinforcing elements in composites and for the fabrication of flexible electronic devices [17].

\subsubsection{Electrical structure and optical property}

The various electronic properties of TMDs arise from the filling of the non-bonding $d$ bands from the group 4 to group 10 species. When the orbitals are partially occupied, the TMDs display metallic properties, whereas when they are fully occupied, they exhibit semiconducting ones. The influence of the chalcogen atoms on the electronic structure is minor compared with that of the metal atoms, however it is observed that the broadening of the $d$ bands decreases the bandgap by increasing the atomic number of the chalcogen [43]. Table 1 summarize the electronic character of different layered TMDs. The bulk TMDs material has an indirect bandgap according to both the theoretical calculations and experimental results [8-15]. For example, in the case of $\mathrm{MoS}_{2}$, bulk $\mathrm{MoS}_{2}$ exhibits a negligible PL signal, however thinner $\mathrm{MoS}_{2}$ nanosheets shows pronounced emissions at $\sim 670$ and $\sim 627 \mathrm{~nm}$ [11]. It also shown that the PL intensity of inversely dependent on the number of $\mathrm{MoS}_{2}$ layers. Especially, single-layer $\mathrm{MoS}_{2}$ shows very strong PL intensity $[11,13]$.

Lee et al. first reported on the characterization of single and few layer $\mathrm{MoS}_{2}$ nanosheets by Raman spectroscopy and AFM [14]. They clearly observed signal inplane $\left(E_{2 g}^{1}\right)$ and out-of-plane $\left(A_{1 g}\right)$ modes for the $\mathrm{MoS}_{2}$ sample with number of layers varying from 1 to 6 . The frequency of $\mathrm{E}_{2 \mathrm{~g}}^{1}$ decreases and that of $\mathrm{A}_{1 \mathrm{~g}}$ increases with increasing number of $\mathrm{MoS}_{2}$ layers. Figures $3 \mathrm{a}$ and $\mathrm{b}$ show the optical image of the thin $\mathrm{MoS}_{2}$ film on a $\mathrm{SiO}_{2} / \mathrm{Si}$ substrate and AFM height image, respectively, and Figures $3 \mathrm{c}$ and $d$ shows the clear red shift of $E_{2 g}^{1}$ and blue shift of $A_{1 g}$ with increasing number of $\mathrm{MoS}_{2}$ layers. The reason for the opposite direction of the frequency shift is the partially Columbic interaction and possible stackinginduced charge of the intra-layer bonding [14]. Optical 

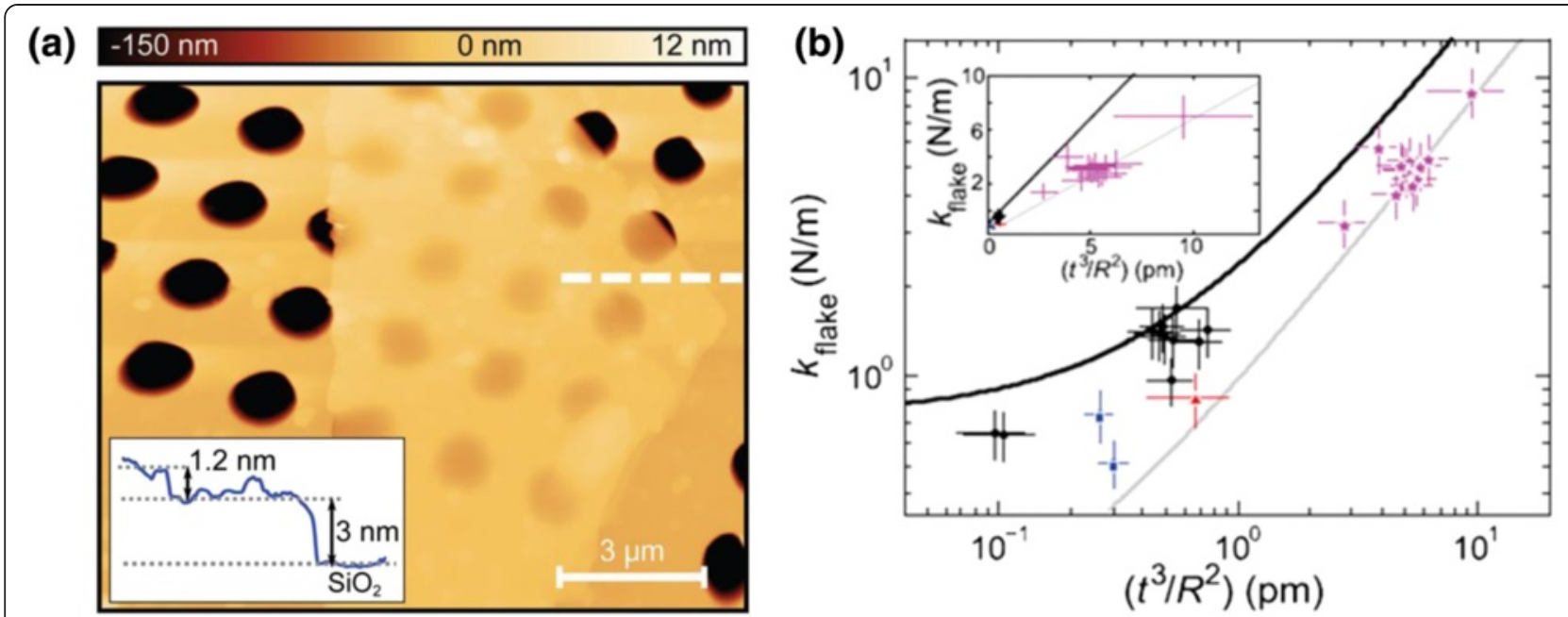

(c)
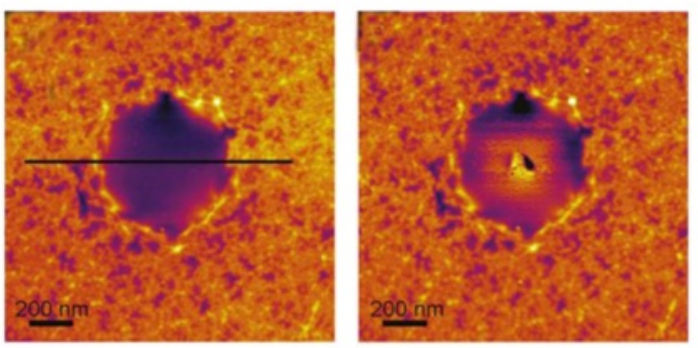

(e)

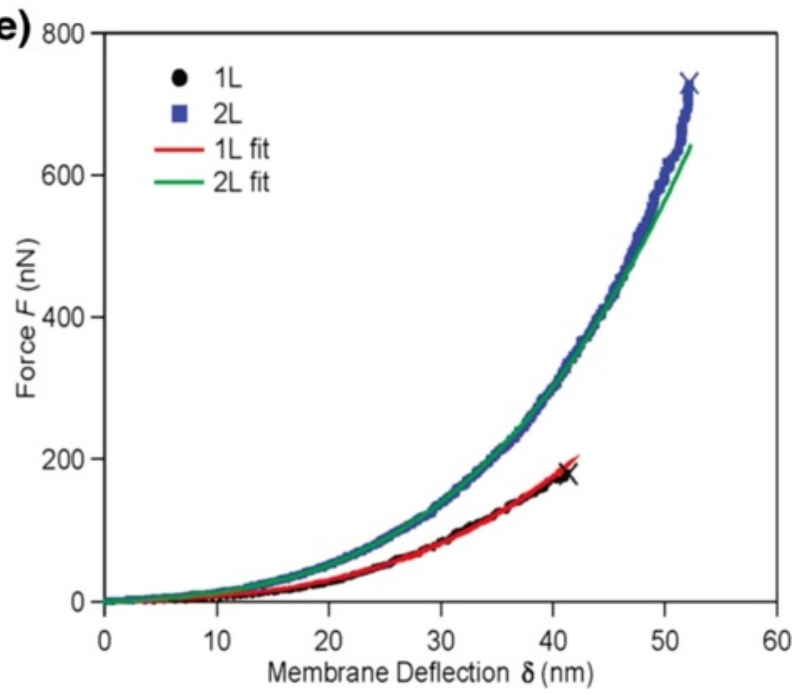

(d)
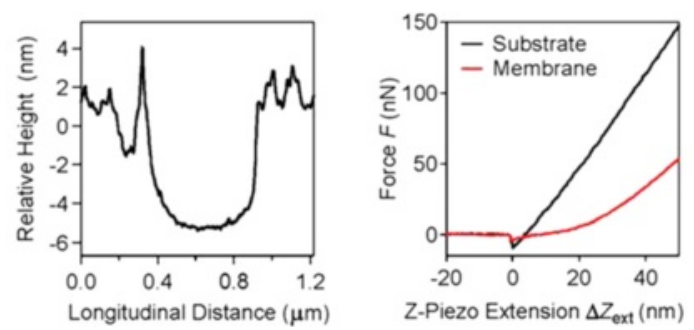

Figure 2 Mechanical propertis of $\mathrm{MoS}_{2}$. (a) AFM topography image of a suspended $5 \sim 7$ layer $\mathrm{MoS}_{2}$ flake on $\mathrm{SiO}_{2} / \mathrm{Si}$ substrate [16]. (b) Elastic constant versus $\mathrm{t}^{3} \mathrm{R}^{-2}$ measured for 26 layer $\mathrm{MoS}_{2}$. The inset graph shows the same graph on a linear scale [16]. (c) AFM topography image of suspended $\mathrm{MoS}_{2}$ membranes before (left) and after (right) the experiment [17]. (d) The height profile of the membrane highlighted in c shows that adheres to the sidewalls over a vertical distance of the order of $5 \mathrm{~nm}$, resulting in a pretension of between $0.02 \mathrm{and}_{0.1} \mathrm{Nm}^{-1}$ (left). Acquired force versus z-piezo extension curves for the suspended membrane and the substrate (right). (e) Examples of loading curves for single and bilayer $\mathrm{MoS}_{2}$ and the least-squares fit of the experimental indentation curves [17].

Table 1 Electronic character of different layered TMDs [43]

\begin{tabular}{llll}
\hline Group & M & X & Properties \\
\hline 4 & Ti, Hf, Zr & S, S, Te & Semiconducting (Eg=0.2 2 eV) \\
5 & V, Nb, Ta & S, Se Te & Narrow band metals or semimetals \\
6 & Mo, W & S, Se, Te & $\begin{array}{l}\text { Sulfides and Selenides are } \\
\text { semiconducting. Telurides are } \\
\text { semietallic. }\end{array}$ \\
7 & Tc, Re & S, Se, Te & $\begin{array}{l}\text { Small gap semiconductors. } \\
\text { Sulfides and Selenides are } \\
10\end{array}$ \\
Pd, Pt & S, Se, Te & $\begin{array}{l}\text { semiconducting. Telurides are } \\
\text { metallic. PdTe } 2 \text { is superconducting }\end{array}$ \\
\hline
\end{tabular}

absorption is another important characteristic of TMDs materials and is related to the band structure of these semiconducting layered materials. Two main peaks can be observed for $\mathrm{Si} / \mathrm{SiO}_{2}$-supported $\mathrm{MoS}_{2}$ at $1.85 \mathrm{eV}$ $(670 \mathrm{~nm})$ and $1.98 \mathrm{eV}(627 \mathrm{~nm})$, respectively. The contrast of the $\mathrm{MoS}_{2}$ layer on a $\mathrm{Si} / \mathrm{SiO}_{2}$ substrate is generally related to the reflective index and absorption contrast of the $\mathrm{MoS}_{2}$ and $\mathrm{SiO}_{2}$ layers. Benameur et al. reported that they were able to distinguish single-, bi-, and tri-layer $\mathrm{MoS}_{2}$ and $\mathrm{WSe}_{2}$ on a 90 or $270 \mathrm{~nm} \mathrm{SiO}_{2}$ substrate by measuring the contrast under broadband green illumination [44]. Recently, Li et al. demonstrated a simple approach that can be used to identify singlelayer to tri-layer $\mathrm{MoS}_{2}$ nanosheets on a $300 \mathrm{~nm} \mathrm{SiO}_{2}$ 

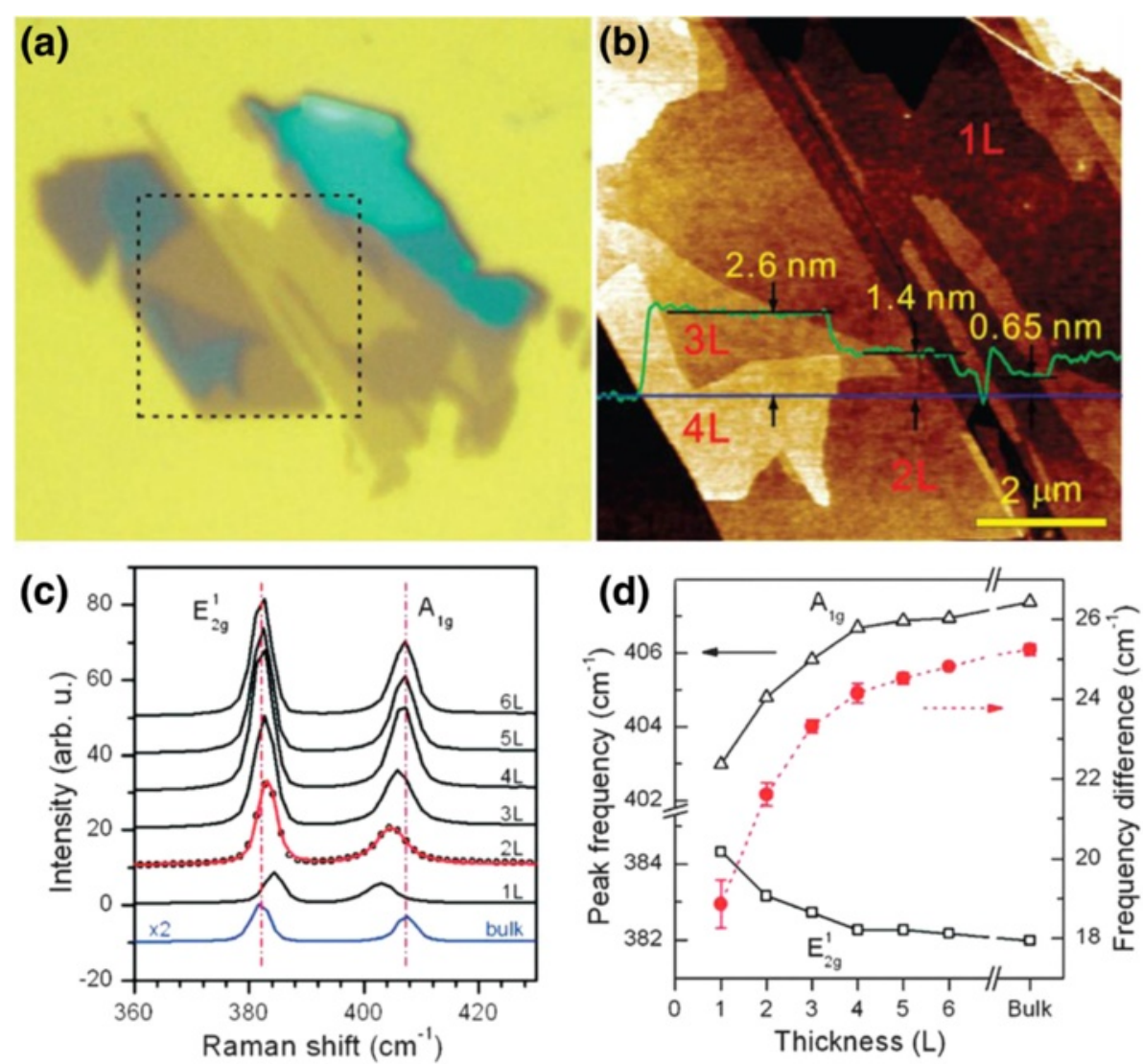

Figure 3 Optical properties of $\mathrm{MoS}_{2}$. (a) Optical micrograph of thin $\mathrm{MoS}_{2}$ films on $\mathrm{SiO}_{2} / \mathrm{Si}$ substrate. [14] (b) AFM height image taken for the 8 $\times 8 \mathrm{~mm}^{2}$ area indicated by dotted lines in (a) [14]. (c) Raman spectra of thin and bulk MoS films. The solid line for the $2 \mathrm{~L}$ spectrum is a double Voigt fit through the data [14]. (d) Frequencies of $E_{2 g}^{1}$ and $A_{1 g}$ Raman modes (left vertical axis) and their difference (right vertical axis) as a function of layer thickness [14].

substrate by the normal optical microscopy imaging method with Image $J$ software [15]. The grayscale image of the $\mathrm{R}$ channel shows distinct layers ranging from single-layer to tri-layers of $\mathrm{MoS}_{2}$ nanohseets. Also, the intensity difference between the $\mathrm{MoS}_{2}$ nanosheets and $\mathrm{SiO}_{2}$ can be used to distinguish the number of $\mathrm{MoS}_{2}$ layers. The direct identification method is a very simple and non-destructive technique for distinguishing the number of $\mathrm{MoS}_{2}$ layers and $\mathrm{MoS}_{2}$ based devices.

\subsection{Synthesis method of TMDs}

\subsubsection{Mechanical exfoliation method}

In 2004, Novoselov et al. successfully produced various single-layer 2D crystals from bulk materials, such as graphite, $\mathrm{BN}, \mathrm{MoS}_{2}, \mathrm{NbSe}_{2}$, and $\mathrm{Bi}_{2} \mathrm{Sr}_{2} \mathrm{CaCu}_{2} \mathrm{O}_{\mathrm{x}}[1,2,45]$. Figures $4 \mathrm{a}$ and $\mathrm{b}$ show the optical and AFM images of the atomically thin $2 \mathrm{D}$ material prepared by the mechanical exfoliation method. This method is typically adopted to prepare single-layer TMDs samples. The single crystal TMDs samples prepared by the mechanical exfoliation method are of good quality, and can be used for studying their basic properties $[26,30,46]$ by optical microscopy, atomic force microscopy (AFM), scanning tunneling microscopy (STM), transmission electron microscopy (TEM) and so on. However, the size of the TMDs material prepared by the mechanical exfoliation method is quite small approximately on the tens of microns scale, posing a limitation to real device applications.

\subsubsection{Liquid exfoliation method}

To exploit the extraordinary potential of these layered materials, large quantities of TMDs nanosheets are required. To obtain large amounts of single- or few-layer TMDs nanosheets, a solution processing strategy would be more appropriate. The first report on the liquid phase exfoliation of sheets of clay materials in the early 1960s [47] has inspired many studies into methods of exfoliating sheets of TMDs [48-52].

Seo et al. reported an interesting sulfidation-induced shape transformation process for the fabricationof $2 \mathrm{D}$ $\mathrm{WS}_{2}$ sheets from $1 \mathrm{D} \mathrm{W}{ }_{18} \mathrm{O}_{49}$ nanorods [53]. The resulting single sheets of $2 \mathrm{D} \mathrm{WS}$ can further assemble together via van der Waals interactions to form nanosheets containing a number of layers. 


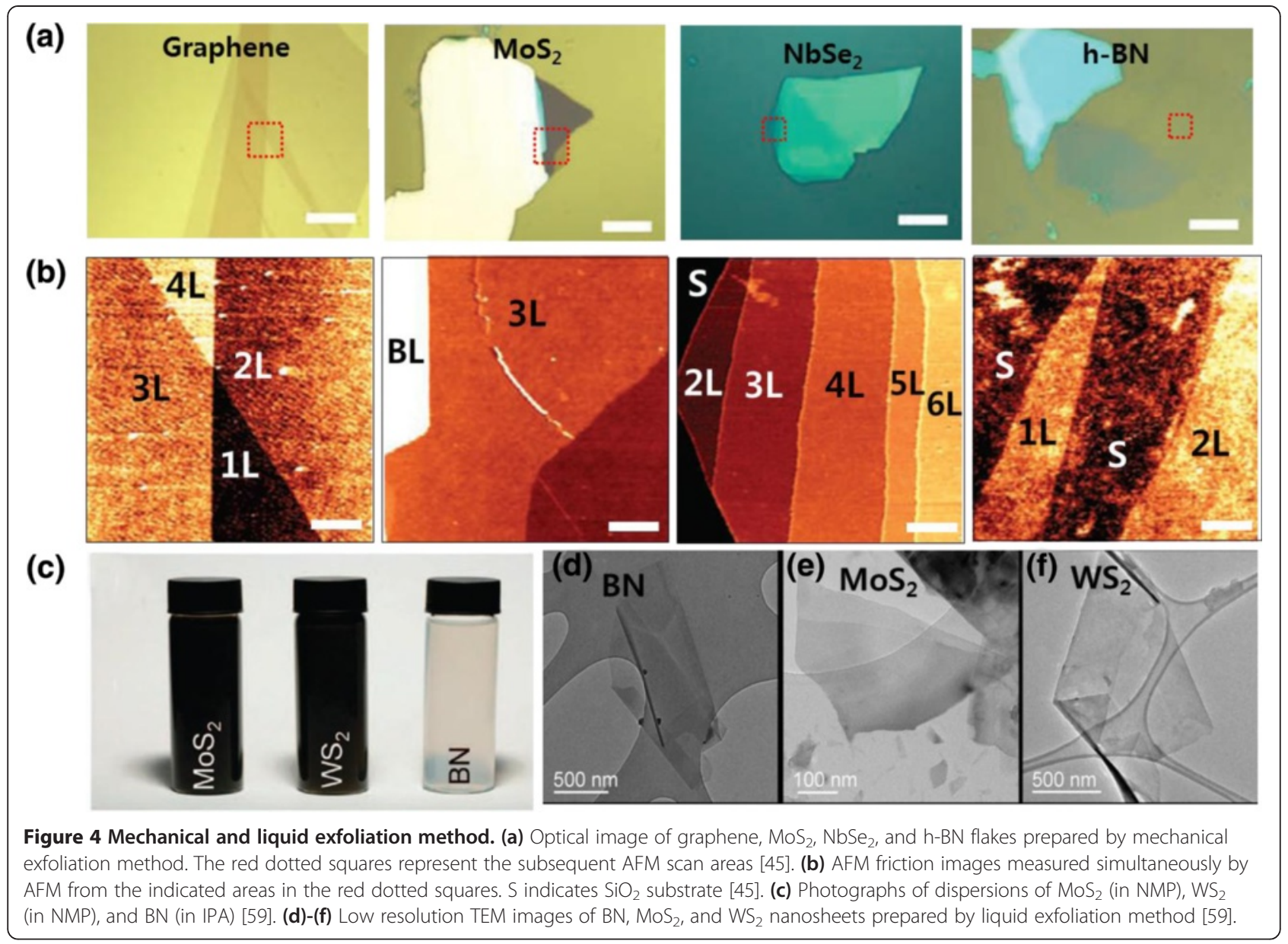

However, the lateral dimension of the $\mathrm{WS}_{2}$ sheets is restricted by the size of the rods. Due to their layered structures, TMDs bulk materials can be intercalated by various kinds of intercalates such as organic molecules, transition metal halides and lithium ions [50]. The resulting intercalated compounds can be exfoliated to single and few-layer 2D TMDs nanosheets by ultrasonication [54-58]. For example, Ramakrishna Matte et al. reported the insertion of $\mathrm{MoS}_{2}$ and $\mathrm{WS}_{2}$ with lithium by using $\mathrm{n}$-butyllithium in hexaneas the intercalation agent, and subsequent exfoliation in water with ultrasonication to yield single-layer materials [54]. However, this method is time-consuming and the degree of lithium insertion is not controllable, which limits it feasibility. Zheng et al. developed a controllable electrochemical lithiation method to produce high-yield, single-layer TMDs nanosheets [30]. By incorporating the layered TMDs bulk materials, such as $\mathrm{MoS}_{2}, \mathrm{WS}_{2}, \mathrm{TiS}_{2}$, and $\mathrm{ZrS}_{2}$, as the cathode in an electrochemical cell, the lithium intercalation in these materials can be monitored and finely controlled during the discharge process. The obtained intercalated compounds can be ultrasonicated and exfoliated in water or ethanol to achieve high-grade TMDs single-layer materials in large amounts.

In another work, Coleman et al. developed an effective and reliable liquid exfoliation technique to produce $2 \mathrm{D}$ nanosheets, including single-layers [59]. After the dispersion and ultrasonication of each inorganic starting material in about 30 common solvents with varying surface tensions and adsoption properties, it was demonstrated that the best solvents have a surface tension close to $40 \mathrm{~mJ} \mathrm{~m} \mathrm{~m}^{-2}$ by using optical absorption spectroscopy. Based on their theoretical investigation, Coleman et al. proposed that successful solvents are those that minimize the energy of exfoliation. For example, N-methyl-pyrrolidone (NMP) and isopropanol (IPA) are very promising solvents for exfoliating various layeredcompounds. The TEM images in Figures $4 \mathrm{c}$ and $\mathrm{d}$ show that very thin sheets of $\mathrm{MoS}_{2}$ and $\mathrm{WS}_{2}$, are produced with lateral sizes ranging from 50 to $1000 \mathrm{~nm}$. These images and associated Fourier transforms illustrate that no substantial deviation from the hexagonal symmetry of these materials is observed, unlike the $\mathrm{MoS}_{2}$ and $\mathrm{WS}_{2}$ nanosheets exfoliated by lithium intercalation $[27,60]$. 


\subsubsection{Sulfurization (or selenization) of metal (or metal oxide) thin film}

To apply TMDs materials to real devices, their large scale growth is essential. The chemical vapor deposition (CVD) method is the most effective way to achieve large-area growth. This method can be divided into two tyes, the sulfurization (or selenization) of metal thin films and vapor phase reaction of metal oxides with chalcogen precursor. Attempts to synthesis $\mathrm{MoS}_{2}$ layers by the simple sulfurization of Mo metal thin films have been reported. Zhan et al. report $\mathrm{MoS}_{2}$ film synthesis by thermal annealing in a sulfur atmosphere with a Mo thin film deposited on a $\mathrm{SiO}_{2} / \mathrm{Si}$ substrate [61]. The size and thickness of the predeposited Mo film determine the size and thickness of the $\mathrm{MoS}_{2}$ thin film, respectively. The direct sulfurization of the Mo metal thin film provides a quick and easy way to access atomically thin $\mathrm{MoS}_{2}$ layers on insulating substrates. However, it is challenging to deposit a uniform Mo film. Kong et al. also reported that vertically aligned $\mathrm{MoS}_{2}$ and molybdenum diselenide (MoSe2) layers can be produced by a rapid sulfurization/selenization process at $550^{\circ} \mathrm{C}$ [62]. Uniform TMDs edge-terminated films with densely packed, strip-like grains can be produced on various substrates including glassy carbon, quartz and oxidized silicon. However, the uncontrolled sulfur/selenium diffusion poses a limitation on the growth process. Further, the anisotropic structure of the TMDs layers makes it much faster for sulfur/selenium diffusion along the van der Waals gaps. Alternatively, attempts have also been made to prepare wafer-scale semiconducting $\mathrm{MoS}_{2}$ thin layers [63] by the direct sulfurization of an $\mathrm{MoO}_{3}$ thin layer (Figures 5a-c). To produce wafer-scale $\mathrm{MoS}_{2}$ (a)

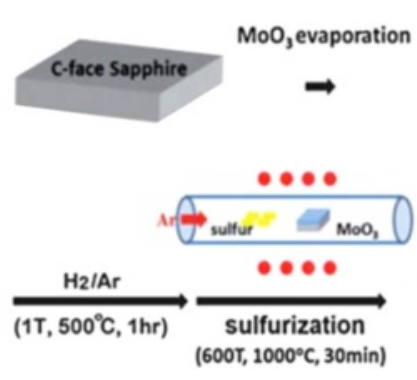

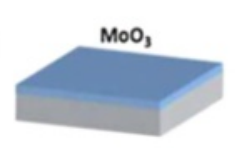

(b)

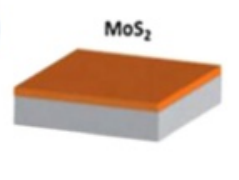

(c)
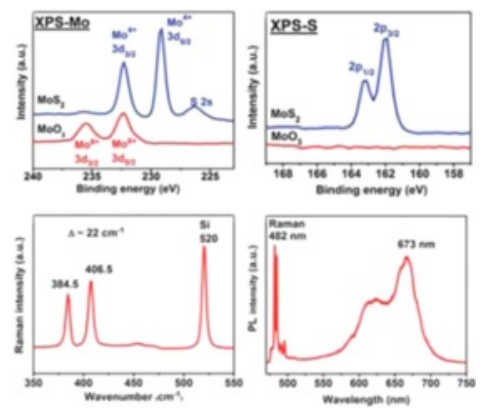

(d)
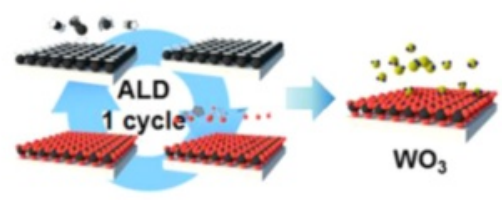

(e)
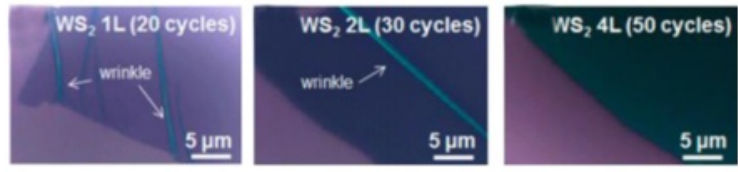

(f)
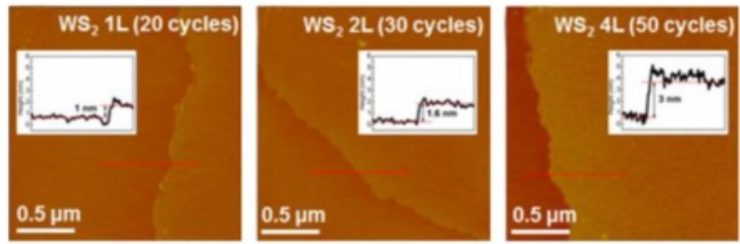

(g)
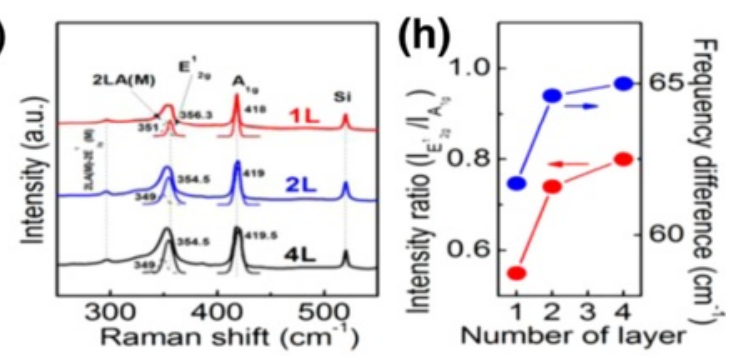

(i)
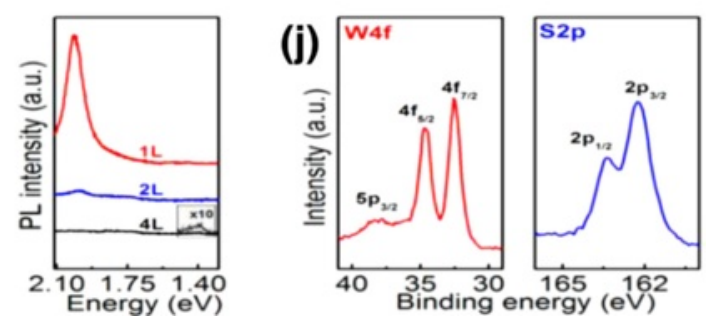

Figure 5 Sulfurization (or selenization) of metal (or metal oxide) thin film. (a) Schematic ilustration for the synthesis of $\mathrm{MoS}_{2}$ layers by $\mathrm{MoO}_{3}$ sulfurization. A layer of $\mathrm{MoO}_{3}(3.6 \mathrm{~nm})$ was thermally evaporated on the sapphire substrate. The $\mathrm{MoO}_{3}$ was then converted to MoS by a two-step thermal process [63]. (b) MoS 2 layer grown on a sapphire wafer [63]. (c) X-Ray photoemission spectroscopy (XPS) results for Mo and S binding energies of the $\mathrm{MoO}_{3}$ layer before and after sulfurization. Raman (lower left) and PL spectra (lower right) for the obtained $\mathrm{MoS}_{2}$ trilayer after $\mathrm{MoO}_{3}$ sulfurization [63]. (d) Synthesis procedure for the ALD-based WS $\mathrm{S}_{2}$ nanosheets [65]. (e) OM images of the transferred WS $\mathrm{S}_{2}$ nanosheet on the $\mathrm{SiO}_{2}$ substrate for the single-, bi-, and tetralayered thicknesses [65]. (f) AFM images and height profiles (inset) of the WS $\mathrm{Wanosheet}_{2}$ transferred onto the $\mathrm{SiO}_{2}$ substrate for the single-, bi-, and tetralayered thicknesses [65]. (g) Raman spectra for the single- (red), bi- (blue), and tetralayer (black) $\mathrm{WS}_{2}$ nanosheets on $\mathrm{SiO}_{2}$ substrates [65]. (h) Relative Raman peak intensities (red) and peak distances (blue) of the $\mathrm{E}_{2 \mathrm{~g}}^{1}$ and $\mathrm{A}_{1 \mathrm{~g}}$

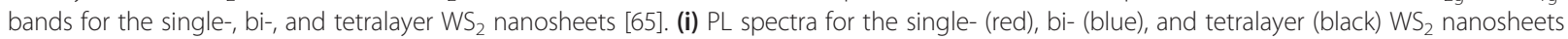
on $\mathrm{SiO}_{2}$ substrates [65]. (j) XPS measurements for the W4f (red) and S2p (blue) core levels of the single-layer $\mathrm{WS}_{2}$ nanosheet [65]. 
thin films, an $\mathrm{MoO}_{3}$ thin layer with the desired thickness is prepared by thermal evaporation on a sapphire substrate. During the growth, an $\mathrm{MoO}_{3}$-coated sapphire substrate is initially reduced to $\mathrm{MoO}_{2}$ or other reduced Mo form in an $\mathrm{H}_{2} / \mathrm{Ar}$ environment at $500^{\circ} \mathrm{C}$. The sample is then annealed in a sulfur-rich environment at $1000^{\circ} \mathrm{C}$. The as-grown $\mathrm{MoS}_{2}$ thin film can be transferred to an arbitrary substrate for the fabrication of electronic devices [63]. A similar technique has also been adopted for the synthesis of large-area $\mathrm{WS}_{2}$ sheets with controllable thickness [64]. However, the synthesis of TMDs by the direct sulfurization (or selenization) of a metal oxide thin film has several limitations, such as the difficulty to control the thickness of the pre-deposited metal oxide or metal thin film, which affects the wafer-scale uniformity. To obtain high quality TMDs with the desired number of layers, the thickness of the metal oxide needs to be precisely controlled. Recently attempts have been made to improve the synthetic process by depositing metal oxide layers via atomic layer deposition (ALD) [65]. A atomically thin TMDs nanosheets with systematic thickness controllability and wafer-scale uniformity can be achieved using this method (Figures 5d and e). Song et al. demonstrated that the number of tungsten disulfide $\left(\mathrm{WS}_{2}\right)$ layers can be controlled by tuning the number of cycles of ALD of tungsten trioxide $\left(\mathrm{WO}_{3}\right)$ [65].

Wang et al. reported a method capable of producing highly crystalline $\mathrm{MoS}_{2}$ flakes with a controlled number of layers by using $\mathrm{MoO}_{2}$ microcrystals as the template [66]. In this method, $\mathrm{MoO}_{2}$ nanoplates are synthesized by the thermal evaporation of $\mathrm{MoO}_{3}$ powder in a sulfur environment at $650-850^{\circ} \mathrm{C}$. The surface of the $\mathrm{MoO}_{2}$ plates is further sulfurized to $\mathrm{MoS}_{2}$ at a higher temperature $\left(850-950^{\circ} \mathrm{C}\right)$ at a later stage. The surface sulfurization of the crystalline $\mathrm{MoO}_{2}$ microplates produces a top $\mathrm{MoS}_{2}$ layer with a high degree of crystallinity However, the $\mathrm{MoS}_{2}$ growth is still determined by the crystal size of the $\mathrm{MoO}_{2}$ flakes, where the $\mathrm{MoS}_{2}$ single crystal obtained is randomly distributed as an isolated island.

\subsubsection{Vaporization of metal oxide with chalcogen precursor}

The very first work on the large area growth of $\mathrm{MoS}_{2}$ atomic layers was reported by Li's group based on the direct chemical vapor phase reaction of $\mathrm{MoO}_{3}$ and $\mathrm{S}$ powders $[67,68]$. During the $\mathrm{MoS}_{2}$ growth, $\mathrm{MoO}_{3}$ in the vapor phase undergoes a two-step reaction, the first of which involves the formation of $\mathrm{MoO}_{3-x}$ that further reacts with the sulfur vapor to grow $\mathrm{MoS}_{2}$ layers. The growth of singlecrystalline $\mathrm{MoS}_{2}$ flakes directly on arbitrary substrates is quite possible by this method and, hence, it has been widely used for producing synthetic TMDs single-layers. The growth of $\mathrm{MoS}_{2}$ is very sensitive to the substrate treatment prior to the growth [67].
Facilitating the nucleation by seeding the substrate with graphene-like species has also been explored [69-71]. Figures $6 \mathrm{a}$ and $\mathrm{b}$ shows the growth setup and condition of $\mathrm{MoS}_{2}$ using $\mathrm{MoS}_{2}$ powder. Up to $400 \mu \mathrm{m}^{2}$ single-layer $\mathrm{MoS}_{2}$ flakes with a triangular shape can be formed on $\mathrm{SiO}_{2}$, sapphire, and glass substrates [70].

Najmaei et al. used $\mathrm{MoO}_{3}$ nanoribbons and sulfur as the reactants for $\mathrm{MoS}_{2}$ growth [72]. The triangular-shaped $\mathrm{MoS}_{2}$ crystals are observed to be nucleated and formed on the step edges. Using substrate patterning by lithography processes, the nucleation of the $\mathrm{MoS}_{2}$ layers can be controlled. The observed catalytic process along the edges is due to the significant reduction in the nucleation energy barrier of $\mathrm{MoS}_{2}$ at the step edges as compared with the flat surface [72]. Further experiments revealed that small triangular $\mathrm{MoS}_{2}$ domains are preferentially nucleated at the step edges and then continue to grow and form boundaries with other domains. Their coalescence finally results in the formation of a continuous $\mathrm{MoS}_{2}$ film [73]. It is also found that the coalescence of the grains leads to the formation of chemically bounded grain boundaries or the simple joining together of the grains by growing on top of each other, without forming any chemical bonds. Van der Zande et al. reported a refined route for ultra-large $\mathrm{MoS}_{2}$ single crystal growth with solid $\mathrm{MoO}_{3}$ and $S$ precursors [73] The resulting highly crystalline islands of single-layer $\mathrm{MoS}_{2}$ can be up to $120 \mathrm{~mm}$ in lateral size. Neither seeding molecules nor step-edges were used to promote the nucleation of $\mathrm{MoS}_{2}$. Large $\mathrm{MoS}_{2}$ crystalline islands with average sizes between 20 and $100 \mathrm{~mm}$ were obtained by using ultraclean substrates and fresh precursors. According to report, the yield can be significantly decreased if dirty substrates or old precursors are used.

The direct sulfurization/selenation of various metal oxides or metal chlorides [74] has been widely applied by many research groups to produce TMDs layers such as $\mathrm{MoS}_{2}$ [74,75], $\mathrm{WS}_{2}$ [64,76,77], $\mathrm{MoSe}_{2}$ [78,79] and $\mathrm{WSe}_{2}$ [80]. Among the numerous reports on the synthesis of sulfides and selenides, one should notice that for the synthesis of selenide, $\mathrm{H}_{2}$ gas is commonly introduced as an additional reducing agent along with Se to further reduce the metal oxides and assist in the selenization reaction $[77,80]$. Furthermore, Zhang et al. revealed that using $\mathrm{H}_{2}$ as the minor carrier gas can also tailor the shape of single-layer $\mathrm{WS}_{2}$ from jagged to straight edge triangles under low pressure chemical vapor deposition [77]. In Figure 6d, Wang et al. demonstrated the CVD growth of uniform $\mathrm{MoSe}_{2}$ single-layer under ambient pressure, resulting in large area single crystalline flakes with a size of $135 \mu \mathrm{m}$. They used $\mathrm{MoO}_{3}$ powder and Se pellets with $\mathrm{Ar} / \mathrm{H}_{2}$ carrier gas. The grown $\mathrm{MoSe}_{2}$ has a direct band gap of $1.48 \mathrm{eV}$, average mobility of $50 \mathrm{~cm}^{2} \mathrm{~V}^{-1} \mathrm{~s}^{-1}$ and on/off ratio of $10^{6}$. 


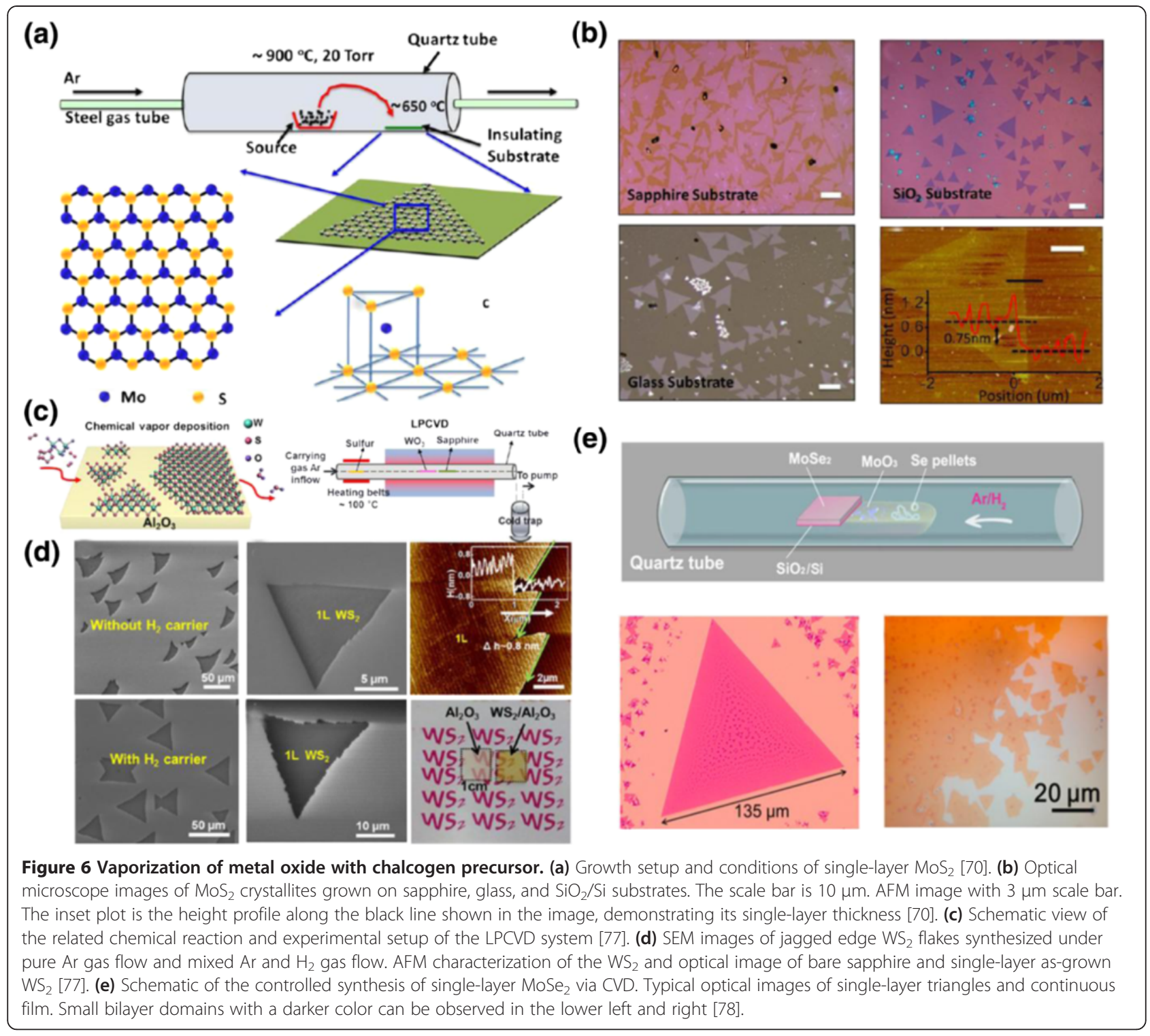

\subsection{Applications of TMDs}

\subsubsection{Electronic devices}

Due to its excellent semiconducting properties with a direct bandgap of $1.83 \mathrm{eV}$, single-layer $\mathrm{MoS}_{2}$ nanosheets are seen as one of the most appropriate supplementing materials to graphene for the fabrication of low power electronic devices. It has been experimentally observed that back-gate transistors based on $\mathrm{MoS}_{2}$ nanosheets with $\mathrm{SiO}_{2}$ dielectric exhibit low carrier mobilities of less than $10 \mathrm{~cm}^{2} \mathrm{~V}^{-1} \mathrm{~s}^{-1}[2,6]$, however, this value can be significantly increased by using a buffer layer of dielectric materials such as $\mathrm{HfO}_{2}$. Thus, the carrier mobility of $\mathrm{MoS}_{2}$ based devices can be increased to over $200 \mathrm{~cm}^{2} \mathrm{~V}^{-1} \mathrm{~s}^{-1}$, along with a large on/off ratio of $1 \times 10^{8}$ and ultralow standby power dissipation at room temperature [6]. The performance limit of $\mathrm{MoS}_{2}$ transistors with $\mathrm{HfO}_{2}$ as the dielectric [20] has been theoretically studied by Yoon et al., who showed that an On/Off current ratio exceeding $10^{10}$ can be achieved. Furthermore, in Figures $7 \mathrm{a}$ and $\mathrm{b}$, $\mathrm{Pu}$ et al. showed the potential of $\mathrm{MoS}_{2}$ based FETs by fabricating the electric bi-layer transistors (EDLTs, FETs gated by ionic liquids) [21,22]. Through the accumulation of carriers, this technique can produce FETs whose performance is up to 2 orders of magnitude higher than that of conventional FETs (e.g. bottom-gate FETs with $300 \mathrm{~nm}$ $\mathrm{SiO}_{2}$ dielectric). Also, using multi-layer $\mathrm{MoS}_{2}$ instead of single-layer $\mathrm{MoS}_{2}$ offers additional advantages. First of all, the density of states of the multi-layer $\mathrm{MoS}_{2}$ is three times that of the single-layer $\mathrm{MoS}_{2}$ leading to a considerably higher drive current in the ballistic limit. In addition, multiple conducting channels can also be created by the field effect in multi-layer $\mathrm{MoS}_{2}$ leading to an increase in the 
(a)

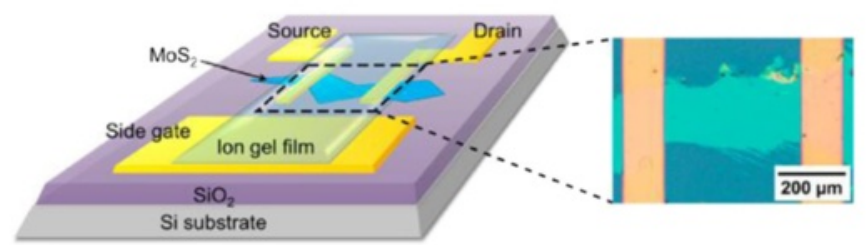

(b)
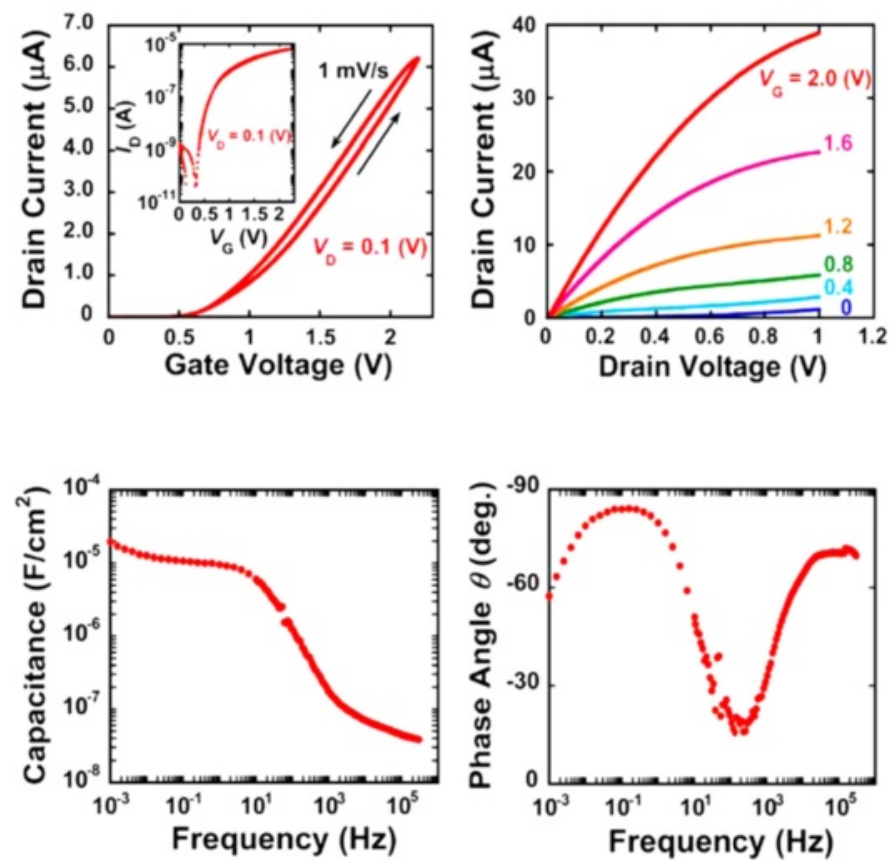

(c)

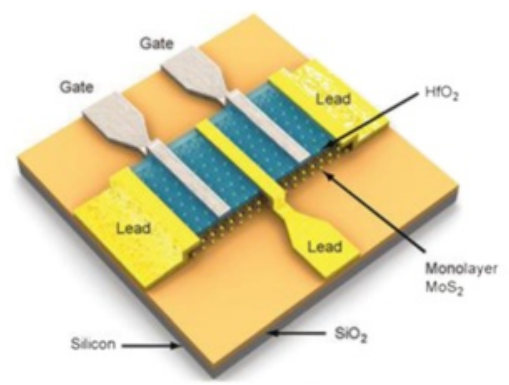

(d)
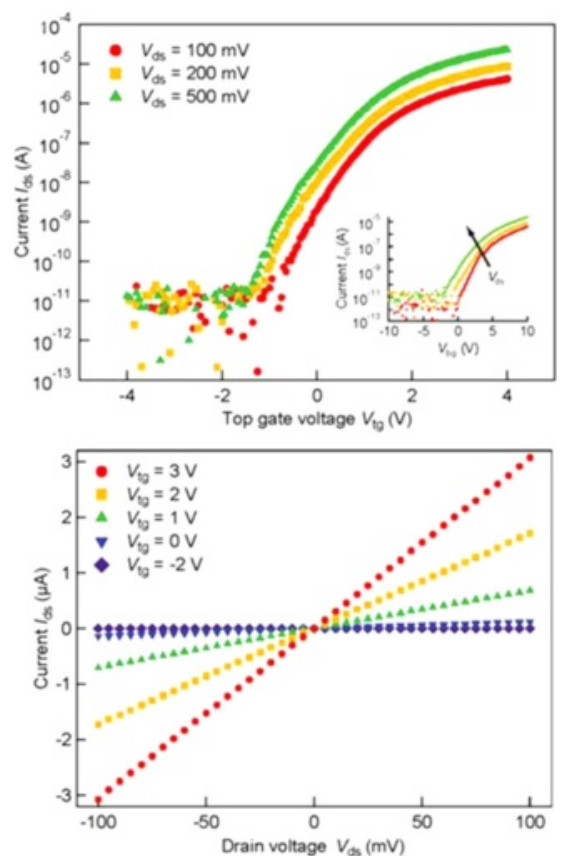

Figure 7 Electronic device applications. (a) A thin film $\mathrm{MoS}_{2}$ EDLT constructed with an ion gel on a rigid substrate [22]. (b) Transfer and output characteristics of the MoS 2 EDLT. $V_{D}$ is the drain voltage, and $V_{G}$ is the gate voltage. Specific capacitance and phase angle of the ion-gel/ $\mathrm{MoS}_{2}$ interface capacitor as a function of the applied frequency [22]. (c) Schematic ilustration for single-layer MoS FET device [24]. (d) Drain-source current $I_{d s}$ through the $\mathrm{MoS}_{2}$ single-layer transistor measured as a function of the top gate voltage $V_{\text {tg }}$ (upper graph). Drain-source current $I_{d s}$ as a function of drain-source voltage for different values of $\mathrm{V}_{\mathrm{tg}}$ (lower graph) [22].

current drive of the device. Radisvljevic et al. experimentally demonstrated that a simple integrated circuit consisting of two $\mathrm{MoS}_{2}$ transistors can be used for amplification as well as performing basic logic operations, as shown in Figures $7 \mathrm{c}$ and $\mathrm{d}$ [24]. The feasibility of incorporating $\mathrm{MoS}_{2}$ transistors into complex circuits was further demonstrated by Wang et al. [25] by integrating 12 transistors side-by-side based on a single sheet of bi-layer $\mathrm{MoS}_{2}$, for various logic operations, including a NAND (Negated AND or NOT AND) gate, astatic random access memory, and a five-stage ring oscillator. Thus, it is expected that semiconducting $\mathrm{MoS}_{2}$ nanosheets can be used as potential building blocks for next generation integrated circuits and various nano-electronic devices.

\subsubsection{Optoelectronic devices}

The thickness dependent band gap of $\mathrm{MoS}_{2}$ nanosheets makes them a potential material for optoelectronic devices.
Recently, single-layer $\mathrm{MoS}_{2}$ based phototransistors were demonstrated, which exhibit an on/off ratio of $\sim 10^{3}$ and a carrier mobility of $0.11 \mathrm{~cm}^{2} \mathrm{~V}^{-1} \mathrm{~s}^{-1}$ can be used for switching them on and off within ca. $50 \mathrm{~ms}$ [26], which is higher than that of single-layer graphene based devices (tens of picosecond) [81]. Also, the photoresponsivity of the $\mathrm{MoS}_{2}$ phototransistors $\left(7.5 \mathrm{~mA} \mathrm{~W}^{-1}\right)$ is much higher than that of graphene-based devices $\left(1 \mathrm{~mA} \mathrm{~W}^{-1}\right)$ [26], due to the zero bandgap, fast carrier transport and short photocarrier lifetime in the pristine graphene, which leads to the fast recombination of photogenerated carriers. Further, Lee et al. fabricated single-, bi- and triple-layer $\mathrm{MoS}_{2}$ phototransistors in the top-gate configuration, consisting of a transparent $50 \mathrm{~nm}$ thick $\mathrm{Al}_{2} \mathrm{O}_{3}$ dielectric to boost the carrier mobility, e.g. up to $80 \mathrm{~cm} 2 \mathrm{~V}^{-1} \mathrm{~s}^{-1}$ for the single-layer device [28]. The phototransistors based on single-layer (bandgap of $1.82 \mathrm{eV}$ ) and bi-layer (bandgap of $1.65 \mathrm{eV}$ ) $\mathrm{MoS}_{2}$ are promising for greenlight detection, while the 
triple-layer $\mathrm{MoS}_{2}$ phototransistor with a bandgap of $1.35 \mathrm{eV}$ is suitable for the detection of red light. Additionally, $\mathrm{MoS}_{2}$ nanosheets can be utilized in the fabrication of light emitting diodes (LEDs) (Figure 8). Frey et al. utilized a thin film of chemically exfoliated $\mathrm{MoS}_{2}$ sheets as the anode for polymer LEDs (PLEDs) [29]. It was found that the $\mathrm{MoS}_{2}$ sheets acted as hole injectors due to their high work function and the wide-gap $\mathrm{MoO}_{3}$ layers acted as an electron-blocking layer in the LED based on the $\mathrm{MoO}_{3} / \mathrm{MoS}_{2}$ hybrid structure, which (a)
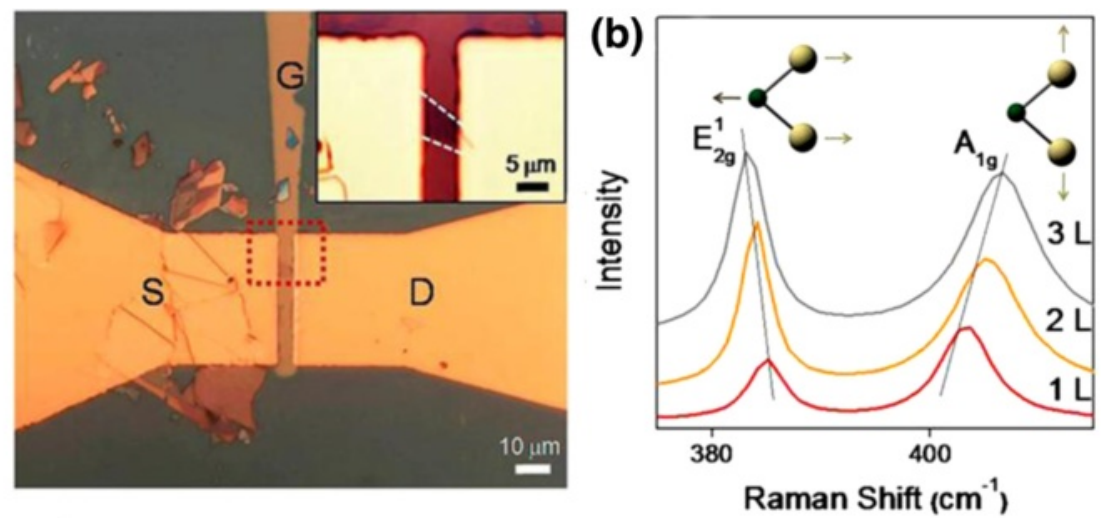

(c)

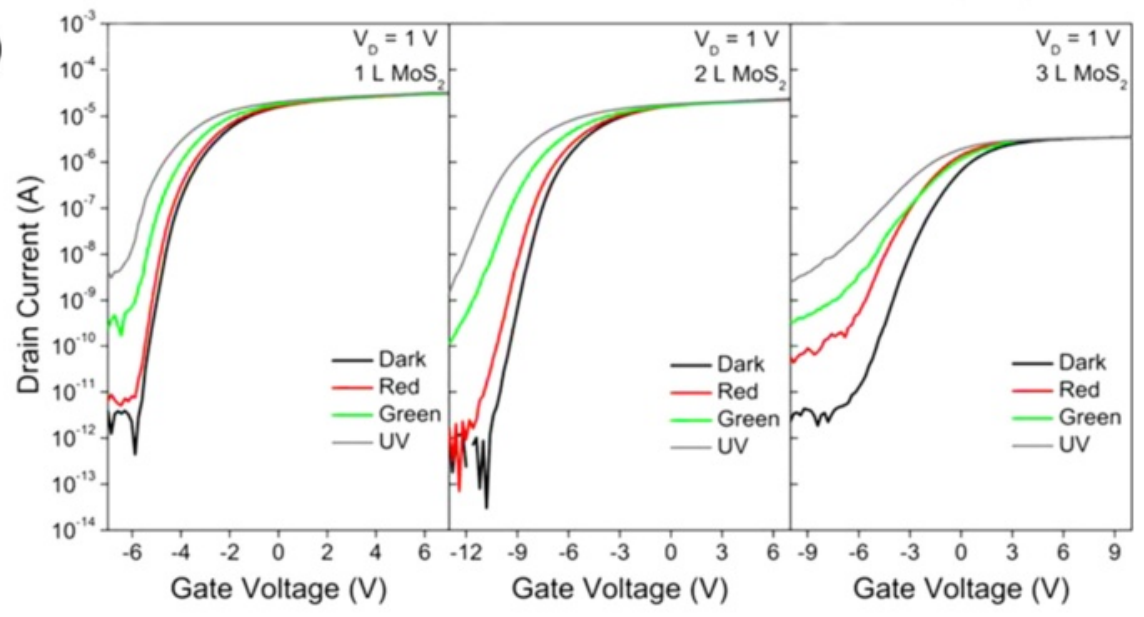

(d)

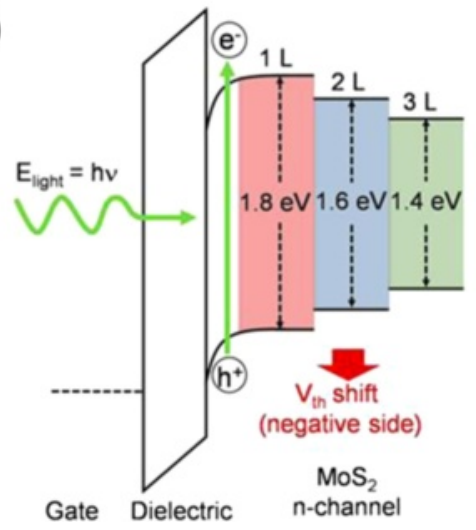

(e)

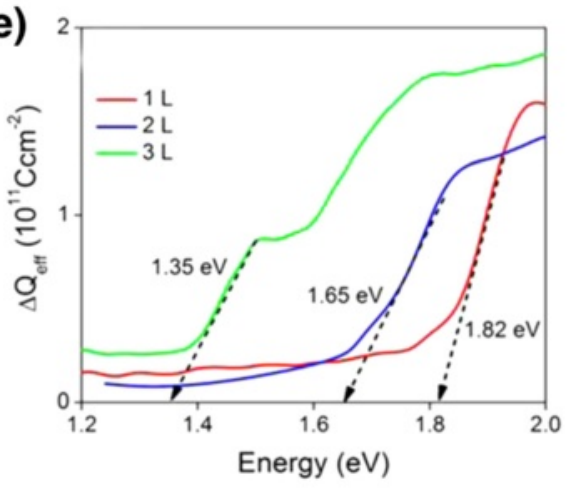

Figure 8 Optoelectronic device applications. (a) Optical image of the single-layer $\mathrm{MoS}_{2}$ based top-gate transistor [28]. (b) Raman spectra of single-, bi-, and triple-layer $\mathrm{MoS}_{2}$. The inset image shows the atomic displacements of the two Raman-active modes: $\mathrm{E}_{2 \mathrm{~g}}^{1}$ and $\mathrm{A}_{1 \mathrm{~g}}$ [28]. (c) The photoinduced transfer curves of respective top-gate transistors with single-, bi-, and ttriple-layer $\mathrm{MoS}_{2}$ under monochromatic red, green, and UV light [28]. (d) The schematic band diagrams of ITO (gate)/ $/ \mathrm{Al}_{2} \mathrm{O}_{3}$ (dielectric)/single (1 L)-, bi (2 L)-, triple (3 L)-layer MoS $(\mathrm{n}$-channel) under the light $($ Elight $=h v)$ illustrate the photoelectric effects for the band gap measurements [28]. (e) The photon energy-dependent $\Delta \mathrm{Q}_{\text {eff }}$ plots indicate the approximate optical energy gaps to be 1.35, 1.65, and $1.82 \mathrm{eV}$ for the triple-, bi-, and single-layer $\mathrm{MoS}_{2}$ nanosheets respectively [28]. 
improved the carrier balance in the device and led to significantly enhanced light emitting performance and efficiency.

\subsubsection{Gas sensing devices}

In recent years, chemical, biological and gas sensors utilizing FET-device structures have become very popular. The change in resistance of the FET channel upon the adsorption of target molecules allows for their detection [32]. Recently, mechanically cleaved single- and multi-layer $\mathrm{MoS}_{2}$ nanosheets based FET devices have been employed for NO detection [30]. It was observed that the FET-based sensors fabricated using bilayer, trilayer, and quadrilayer $\mathrm{MoS}_{2}$ nanosheets exhibited high sensitivity for NO with a detection limit of less than $1 \mathrm{ppm}$ (Figures 9a and b). However FET-based sensing using a single-layer device presented an unstable response. Further, a flexible gas sensor using $\mathrm{MoS}_{2}-\mathrm{rGO}$ hybrid structure was fabricated on a polyethylene terephthalate
(PET) substrate for the detection of $\mathrm{NO}_{2}$ (Figures 9c and d) [33]. In addition, the functionalization of the $\mathrm{MoS}_{2}$ thin film with Pt nanoparticles (NPs) led to an increase in the sensing sensitivity by a factors of 3 , with a detection limit of $\sim 2 \mathrm{ppb}$ for $\mathrm{NO}_{2}$. Also, a systematic study on the electrochemical sensing behavior of solution processable $\mathrm{MoS}_{2}$ nanosheets was reported [34] in which the single-layer $\mathrm{MoS}_{2}$ nanosheets attached to a (3-aminopropyl)-triethoxysilane (APTES)-functionalized glass carbon electrode exhibited a fast electron transfer rate in the $[\mathrm{Fe}(\mathrm{CN}) 6]^{3-/ 4}$-and $[\mathrm{Ru}(\mathrm{NH} 3) 6]^{2+/ 3+}$ redox systems.

\subsubsection{Energy storage devices}

The large-scale production of single- and multi-layer $\mathrm{MoS}_{2}$ nanosheets using exfoliation techniques can enable their wide spread application for energy storage devices such as batteries. Recently, $\mathrm{MoS}_{2}$ nanosheets prepared by chemical lithiation and exfoliation in a stacking structure were fabricated for use as electrodes for lithium ion batteries (LIBs)
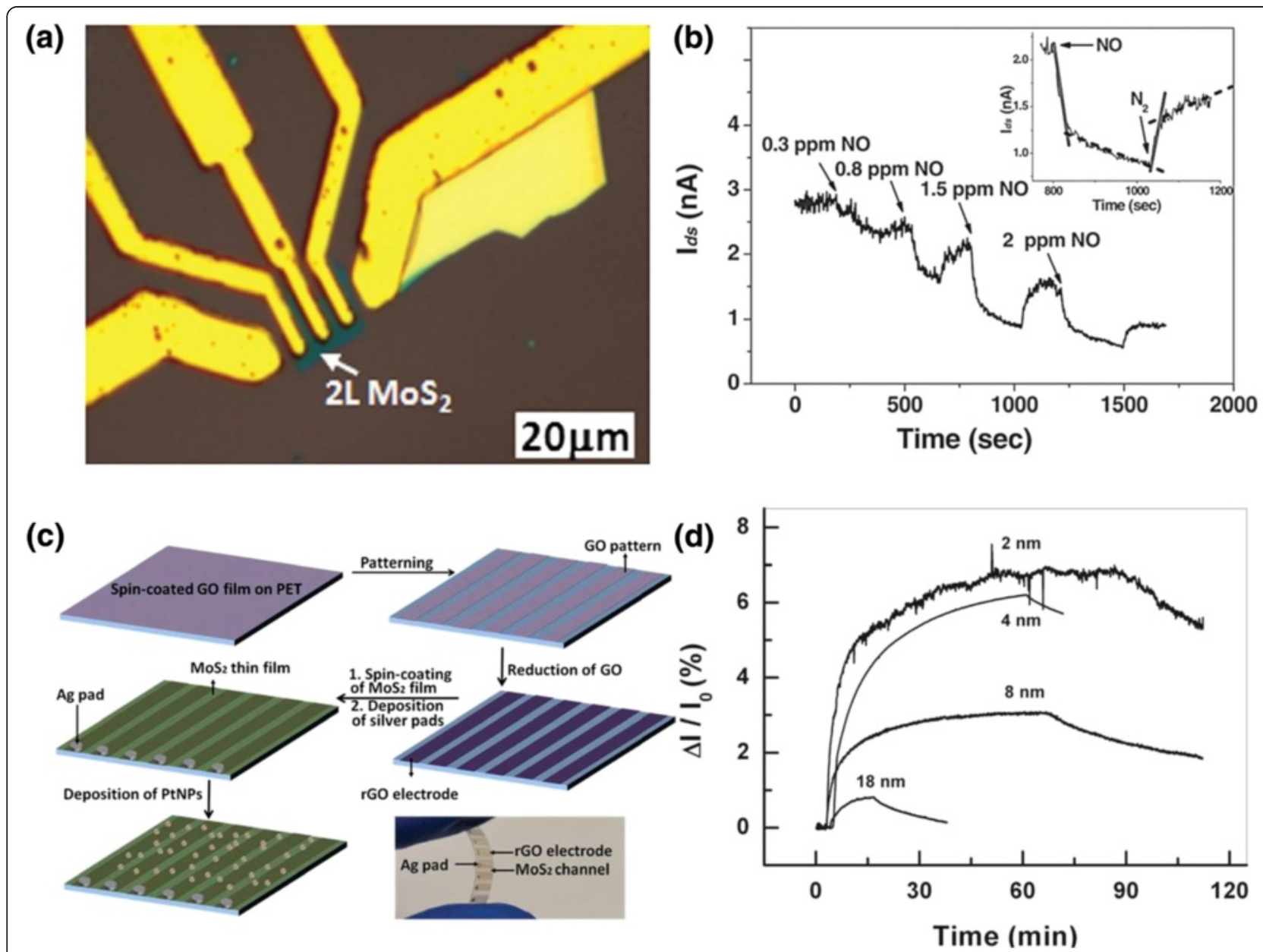

Figure 9 Gas sensing devices application. (a) Optical microscope image of an FET device based on 2 L MoS $_{2}$ [30]. (b) Real-time current response after exposure of the $2 \mathrm{LMOS}_{2} \mathrm{FET}$ to increasing concentrations of NO. Inset: A typical adsorption and desorption process of NO on the $2 \mathrm{LMOS}_{2}$ FET [30]. (c) Schematic illustration of the fabrication process of MoS 2 TFT array on PET substrate and a photograph of the TFT sensor array [33]. (d) Detection of $1.2 \mathrm{ppm} \mathrm{NO} \mathrm{N}_{2}$ using $\mathrm{MoS}_{2}$ TFT sensors on PET with different thicknesses of $\mathrm{MoS}_{2}$ thin film [33]. 
and compared with electrodes made from the bulk $\mathrm{MoS}_{2}$ (Figures 10a and b) [37]. The stacked $\mathrm{MoS}_{2}$ nanostructure showed much better cycling stability than the bulk $\mathrm{MoS}_{2}$, retaining a high capacity of $750 \mathrm{~mA} \mathrm{~h} \mathrm{~g}$ even after 50 cycles. The stability and Li-storage capacity of $\mathrm{MoS}_{2}$ based energy storing devices can be improved to a greater extent by adding polymer molecules such as poly(ethylene oxide)(PEO) to the Li-intercalation solution [38], as the presence of $\mathrm{PEO}$ can increase in the interlayer spacing of the $\mathrm{MoS}_{2}$ nanosheets. Much improved Li-storage capacity and cycling stability are observed in the case of electrodes made from the $\mathrm{MoS}_{2}-\mathrm{PEO}$ nanocomposites. This improvement in performance is attributed to the fact that large amounts of lithium ions can be accommodated over the PEO. Recently, few-layer $\mathrm{MoS}_{2}$ and reduced graphene oxide (rGO) hybrids were used for the fabrication of electrodes that exhibited a specific capacity of
$1100 \mathrm{~mA} \mathrm{~h} \mathrm{~g}^{-1}$ at a current of $100 \mathrm{~mA} \mathrm{~g}^{-1}$ with excellent cycling stability.

The $\mathrm{MoS}_{2}$ nanosheets can be used for the fabrication of supercapacitors or bi-layer capacitors, as they possess a large interlayer space, as well as a large specific surface area, that can be used for ion intercalation, and exhibit several stable oxidation states from $\mathrm{Mo}^{2+}$ to $\mathrm{Mo}^{6+}$. Recently, a thin film $(\sim 100 \mathrm{~nm})$ of CVD-grown edge-oriented $\mathrm{MoS}_{2}$ was employed as a supercapacitor electrode [40] and the as-fabricated supercapacitor exhibited dual functionality, i.e. it exhibits both bi-layer and faradaic capacitance, and can function even at alternating current frequencies of $\sim 100 \mathrm{~Hz}$.

\section{Conclusion}

In summary, we discussed the various properties of TMDs, including their electrical, mechanical, and optical ones. (a)

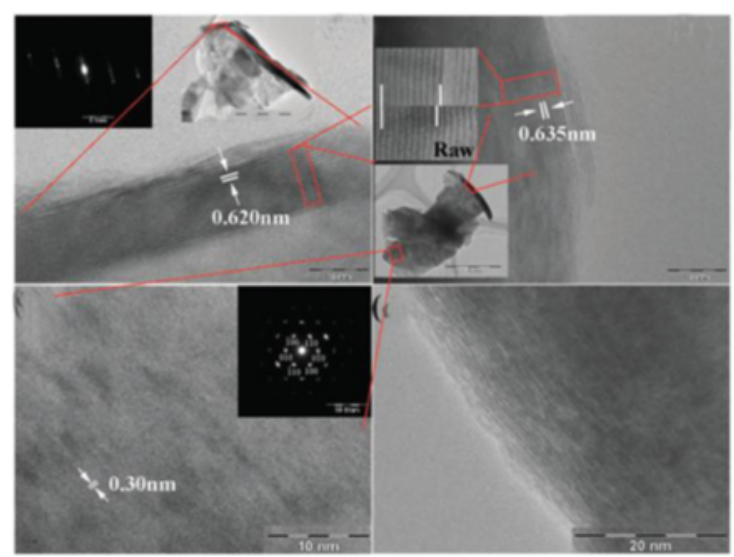

(c)

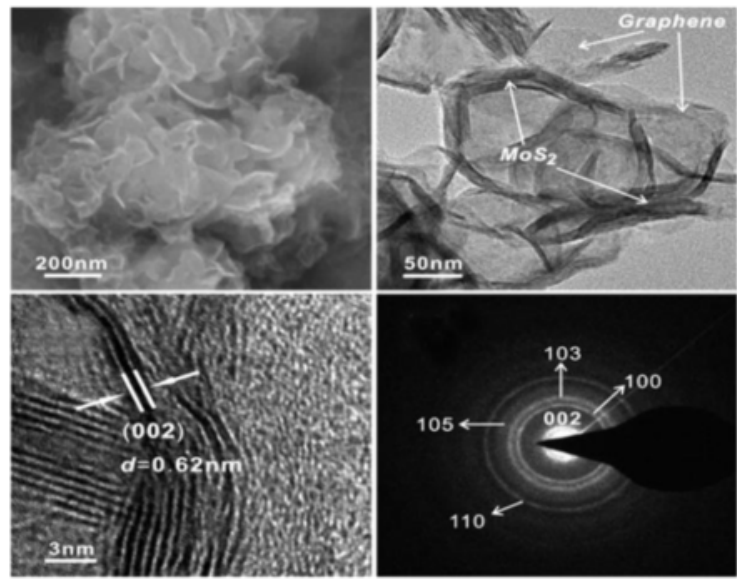

(b)
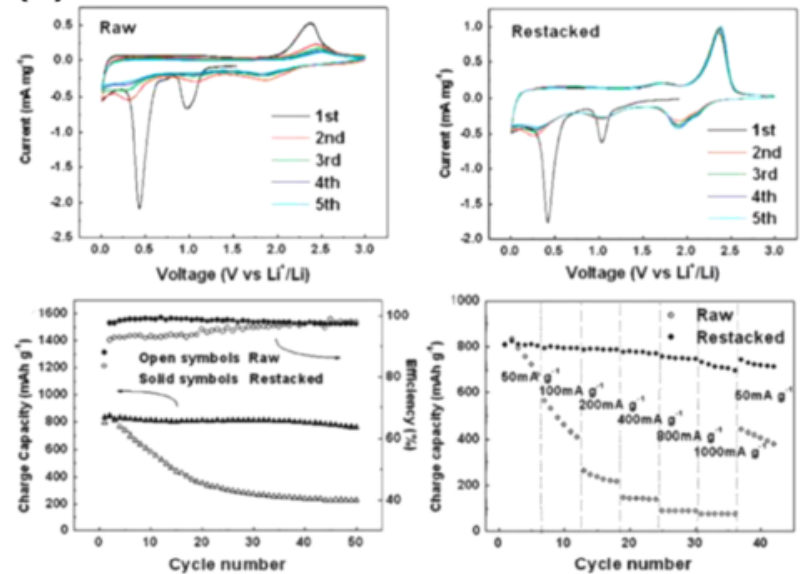

Voltage (V vs Lílu)

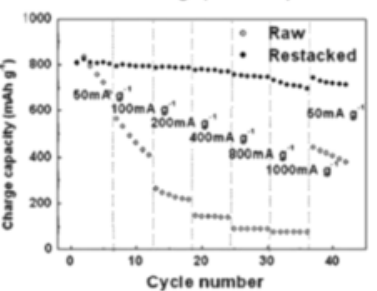

(d)

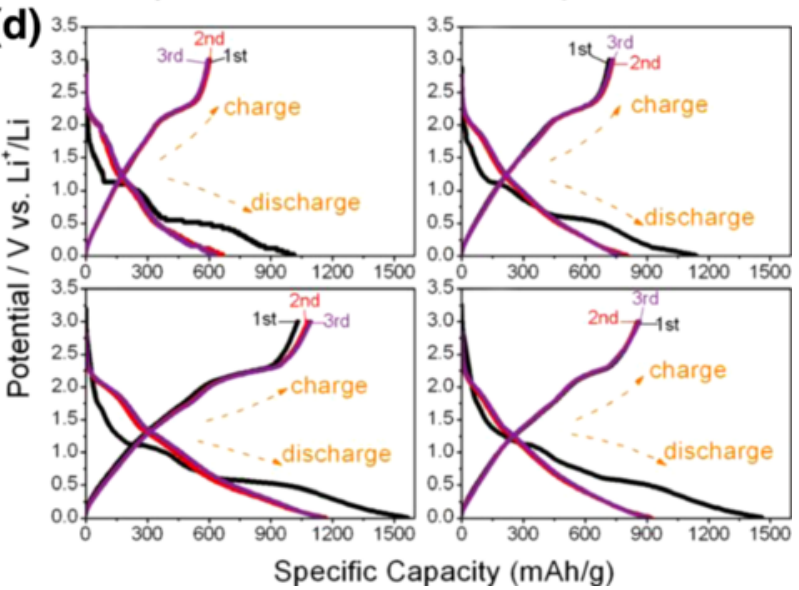

Figure 10 Energy storage device application. (a) TEM images of raw and restacked $\mathrm{MoS}_{2}$ [37]. (b) Electrochemical properties of raw and restacked $\mathrm{MoS}_{2}$ : cyclic voltammograms of raw and restacked $\mathrm{MoS}_{2}$ at a scanning rate of $0.2 \mathrm{mV} \mathrm{s}^{-1}$ [37]. (c) Microstructure of $\mathrm{MoS}_{2} / \mathrm{G}$ (1:2) composite: SEM image, TEM image, HRTEM image, and electron diffraction pattern [40]. (d) First three charge and discharge curves of the samples after annealing in $\mathrm{H}_{2} / \mathrm{N}_{2}$ at $800^{\circ} \mathrm{C}$ for $2 \mathrm{~h}$ at a current density of $100 \mathrm{~mA} / \mathrm{g}$ in a half-cell composed of $\mathrm{MoS}_{2} / \mathrm{G}$ and Li: $\mathrm{MoS}_{2}, \mathrm{MoS}_{2} / \mathrm{G}$ (1:1), $\mathrm{MoS}_{2} / \mathrm{G}(1: 2),(\mathrm{d}) \mathrm{MoS}_{2} / \mathrm{G}(1: 4)[40]$. 
Because of their unique properties, TMDs can be applied in various fields, such as electronics, optoelectronics, sensing and energy storage applications. As a typical example, bulk $\mathrm{MoS}_{2}$ has an indirect band gap of $1.2 \mathrm{eV}$, whereas single-layer $\mathrm{MoS}_{2}$ is a direct band gap semiconductor with a band gap of $1.8 \mathrm{eV}$ due to the quantum confinement effect, which results in the enhancement of its photoluminescence. Further, it is experimentally found that the single-layer $\mathrm{MoS}_{2}$ based FETs exhibit a remarkably high on/off ratio of $\sim 100$ at room temperature. In addition, single-layer $\mathrm{MoS}_{2}$ transistors have been shown to exhibit much better photoresponsivity than graphene based ones. Moreover, gas sensors based on few layer $\mathrm{MoS}_{2}$ nanosheets have shown high sensitivity for NO detection. Also, we discussed the various sample preparation methods. The TMDs samples prepared by mechanical and liquid exfoliation methods have good quality, but their size is small. The CVD approach is suitable for wafer scale fabrication and real device applications.

\section{Competing interests}

The authors declare that they have no competing interests.

\section{Authors' contributions}

$\mathrm{SAH}, \mathrm{RB}$ and S-WK contributed to the preparation of the manuscript. All authors read and approved the final manuscript.

\section{Authors' information}

Sang A Han: Sang A Han is a pursuing her Ph.D. degree under the supervision of Prof. Sang-Woo Kim at SKKU Advanced Institute of Nanotechnology (SAINT), Sungkyunkwan University (SKKU). Her research interests are the synthesis and characterization of two-dimensional materials and their applications. Ravi Bhatia: Ravi Bhatia is working as a Postdoctoral Fellow with Prof. Sang-Woo Kim at Sungkyunkwan University (SKKU), South Korea. He earned his doctorate degree from the Department of Physics, the Indian institute of Science (IISC), in 2012. During his PhD tenure, he worked on the low temperature charge transport and magnetic properties of iron-filled multiwall carbon nanotube (MWCNT) and MWCNT based composite systems. His current research interests are focused on studying various aspects of two dimensional materials.

Sang-Woo Kim: Sang-Woo Kim is an Associate Professor in the School of Advanced Materials Science and Engineering at Sungkyunkwan University (SKKU). He received his Ph.D. from Kyoto University in the Department of Electronic Science and Engineering in 2004. After working as a postdoctoral researcher at Kyoto University and the University of Cambridge, he spent 4 years as an assistant professor at Kumoh National Institute of Technology. He joined the School of Advanced Materials Science and Engineering, Advanced Institute of Nanotechnology (SAINT) at SKKU in 2009. His recent research interests have been focused on piezoelectric/triboelectric nanogenerators, photovoltaics, and two-dimensional nanomaterials including graphene and hexagonal boron nitride nanosheets. $\mathrm{He}$ "' is currently an Associate Editor of Nano Energy and Executive Editorial Board of Advanced Electronic Materials.

\section{Acknowledgements}

This work was financially supported by Basic Science Research Program (2012R1A2A1A01002787, 2009-0083540) and the Center for Advanced Soft-Electronics as Global Frontier Project (2013M3A6A5073177) through the National Research Foundation (NRF) of Korea Grant funded by the Ministry of Science, ICT \& Future Planning.

Received: 5 March 2015 Accepted: 16 March 2015

Published online: 01 September 2015

\section{References}

1. KS Novoselov, AK Geim, SV Morozov, D Jiang, Y Zhang, SV Dubonos, IV Grigorieva, AA Firsov, Science 306, 666 (2004)

2. KS Novoselov, D Jiang, F Schedin, TJ Booth, W Khotkevich, SV Morozov, AK Geim, Proc. Natl. Acad. Sci. U. S. A. 102, 10451 (2005)

3. L Song, L Ci, H Lu, PB Sorokin, C Jin, J Ni, AG Kvashnin, DG Kvashnin, J Lou, BI Yakobson, PM Ajayan, Nano Lett. 10, 3209 (2010)

4. Y Shi, C Hamsen, X Jia, KK Kim, A Reina, M Hofmann, AL Hsu, K Zhang, H Li, ZY Juang, MS Dresselhaus, L Li, J Kong, Nano Lett. 10, 4134 (2010)

5. KH Lee, HJ Shin, JY Lee, IY Lee, GH Kim, JY Choi, SW Kim, Nano Lett. $12,714(2012)$

6. B Radisavljevic, A Radenovic, J Brivio, V Giacometti, A Kis, Nat. Nanotechnol. 6, $147(2011)$

7. O Lopez-Sanchez, D Lembke, M Kayci, A Radenovic, A Kis, Nat. Nanotechnol. 8, 497 (2013)

8. H Zeng, J Dai, W Yao, D Xiao, X Cui, Nat. Nanotechnol. 7, 490 (2012)

9. W Zhang, C-P Chuu, J-K Huang, C-H Chen, M-L Tsai, Y-H Chang, C-T Liang, Y-Z Chen, Y-L Chueh, J-H He, M-Y Chou, L-J Li, Sci. Rep. 4, 3826 (2014)

10. C Cong, J Shang, X Wu, B Cao, N Peimyoo, C Qiu, L Sun, T Yu, Adv. Opt. Mater. 2, 131 (2014)

11. A Splendiani, L Sun, YB Zhang, TS Li, J Kim, CY Chim, G Galli, F Wang, Nano Lett. 10, 1271 (2010)

12. T Li, G Galli, J. Phys. Chem. C 111, 16192 (2007)

13. KF Mak, C Lee, J Hone, J Shan, TF Heinz, Phys. Rev. Lett. 105, 136805 (2010)

14. C Lee, H Yan, LE Brus, TF Heinz, J Hone, S Ryu, ACS Nano 4, 2695 (2010)

15. H Li, G Lu, ZY Yin, QY He, Q Zhang, H Zhang, Small 8, 682 (2012)

16. A Castellanos-Gomez, M Poot, GA Steele, HSJ van der Zant, N Agraït, G Rubio-Bollinger, Adv. Mater. 24, 772 (2012)

17. S Bertolazzi, J Brivio, A Kis, ACS Nano 5, 9703 (2011)

18. X Huang, Z Yin, S Wu, X Qi, Q He, Q Zhang, Q Yan, F Boey, H Zhang, Small 7, $1876(2011)$

19. Y Zhang, T-T Tang, C Girit, Z Hao, MC Martin, A Zettl, MF Crommie, YR Shen, F Wang, Nature 459, 820 (2009)

20. Y Yoon, K Ganapathi, S Salahuddin, Nano Lett. 11, 3768 (2011)

21. YJ Zhang, JT Ye, Y Matsuhashi, Y Iwasa, Nano Lett. 12, 1136 (2012)

22. J Pu, Y Yomogida, K-K Liu, L-J Li, Y Iwasa, T Takenobu, Nano Lett. 12, 4013 (2012)

23. S Kim, A Konar, W-S Hwang, JH Lee, J Lee, J Yang, C Jung, H Kim, J-B Yoo, J-Y Choi, YW Jin, SY Lee, D Jena, W Choi, K Kim, Nat. Commun. 3, 1011 (2012)

24. B Radisavljevic, MB Whitwick, A Kis, ACS Nano 5, 9934 (2011)

25. H Wang, L Yu, Y-H Lee, Y Shi, A Hsu, ML Chin, L-J Li, M Dubey, J Kong, T Palacios, Nano Lett. 12, 4674 (2012)

26. ZY Yin, $H$ Li, L Jiang, YM Shi, YH Sun, G Lu, Q Zhang, XD Chen, H Zhang, ACS Nano 6, 74 (2012)

27. GL Frey, KJ Reynolds, RH Friend, H Cohen, Y Feldman, J. Am. Chem. Soc 125, 5998 (2003)

28. HS Lee, S-W Min, Y-G Chang, MK Park, T Nam, H Kim, JH Kim, S Ryu, S Im, Nano Lett. 12, 3695 (2012)

29. C Zhong, C Duan, F Huang, H Wu, Y Cao, Chem. Mater. 23, 326 (2010)

30. H Li, ZY Yin, QY He, X Huang, G Lu, DWH Fam, AlY Tok, Q Zhang, H Zhang, Small 8, 63 (2012)

31. ZY Zeng, ZY Yin, X Huang, H Li, QY He, G Lu, F Boey, H Zhang Angew, Chem Int. Ed 50, 11093 (2011)

32. Q He, S Wu, Z Yin, H Zhang, Chem. Sci. 3, 1764 (2012)

33. Q He, Z Zeng, Z Yin, H Li, S Wu, X Huang, H Zhang, Small 8, 2994 (2012)

34. S Wu, Z Zeng, Q He, Z Wang, SJ Wang, Y Du, Z Yin, X Sun, W Chen, H Zhang, Small 8, 2264 (2012)

35. MR Palacin, Chem. Soc. Rev. 38, 2565 (2009)

36. MS Whittingham, Chem. Rev. 104, 4271 (2004)

37. G Du, Z Guo, S Wang, R Zeng, Z Chen, H Liu, Chem. Commun. 46, $1106(2010)$

38. J Xiao, D Choi, L Cosimbescu, P Koech, J Liu, JP Lemmon, Chem. Mater. $22,4522(2010)$

39. X Huang, X Qi, F Boey, H Zhang, Chem. Soc. Rev. 41, 666 (2012)

40. K Chang, WX Chen, ACS Nano 5, 4720 (2011)

41. BE Conway, Electrochemical Supercapacitors: Scientific Fundamentals and Technological Applications (Kulwer Academic/Plenum Press, New York, 1999)

42. JM Soon, KP Loh, Electrochem. Solid-State Lett. 10, A250 (2007)

43. M Chhowalla, HS Shin, G Eda, LJ Li, KP Loh, H Zhang, Nat. Chem. 5, 263 (2013) 
44. MM Benameur, B Radisavljevic, JS He'ron, S Sahoo, H Berger, A Kis, Nanotechnology 22, 125706 (2011)

45. C Lee, QY Li, W Kalb, XZ Liu, H Berger, RW Carpick, J Hone, Science 328, 76 (2010)

46. S Ghatak, AN Pal, A Ghosh, ACS Nano 5, 7707 (2011)

47. GF Walker, Nature 187, 312 (1960)

48. J Feng, X Sun, CZ Wu, LL Peng, CW Lin, SL Hu, JL Yang, Y Xie, J. Am. Chem Soc. 133, $17832(2011)$

49. J Feng, LL Peng, CZ Wu, X Su, SL Hu, CW Lin, J Dai, JL Yang, Y Xie, Adv. Mater. 24, 1969 (2012)

50. MB Dines, J. Chem. Educ. 51, 211 (1974)

51. T Sasaki, M Watanabe, H Hashizume, H Yamada, H Nakazawa, J. Am. Chem. Soc. $118,8329(1996)$

52. D Golberg, Nat. Nanotechnol. 6, 200 (2011)

53. JW Seo, YW Jun, SW Park, H Nah, T Moon, B Park, JG Kim, YJ Kim, J Cheon, Angew. Chem. Int. Ed. 46, 8828 (2007)

54. HSS Ramakrishna Matte, A Gomathi, AK Manna, DJ Late, R Datta, SK Pati, CNR Rao, Angew. Chem. Int. Ed. 122, 4153 (2010)

55. P Joensen, RF Frindt, SR Morrison, Mater. Res. Bull. 21, 457 (1986)

56. BK Miremadi, SR Morrison, J. Appl. Phys. 63, 4970 (1988)

57. D Yang, RF Frindt, J. Phys. Chem. Solids 57, 1113 (1996)

58. G Eda, H Yamaguchi, D Voiry, T Fujita, MW Chen, M Chhowalla, Nano Lett. 11, $5111(2011)$

59. JN Coleman, M Lotya, A O'Neill, SD Bergin, PJ King, U Khan, K Young, A Gaucher, S De, RJ Smith, IV Shvets, SK Arora, G Stanton, HY Kim, K Lee, GT Kim, GS Duesberg, T Hallam, JJ Boland, JJ Wang, JF Donegan, JC Grunlan, G Moriarty, A Shmeliov, RJ Nicholls, JM Perkins, EM Grieveson, K Theuwissen, DW McComb, PD Nellist et al., Science 331, 568 (2011)

60. RA Gordon, D Yang, ED Crozier, DT Jiang, RF Frindt, Phys. Rev. B 65, 125407 (2002)

61. Y Zhan, Z Liu, S Najmaei, PM Ajayan, J Lou, Small 8, 966 (2012)

62. D Kong, H Wang, JJ Cha, M Pasta, KJ Koski, J Yao, Y Cui, Nano Lett. 13, 1341 (2013)

63. Y-C Lin, W Zhang, J-K Huang, K-K Liu, Y-H Lee, C-T Liang, C-W Chu, L-J Li, Nanoscale 4, 6637 (2012)

64. AL Elı'as, N Perea-Lo'pez, A Castro-Beltra'n, A Berkdemir, R Lv, S Feng, AD Long, T Hayashi, YA Kim, M Endo, HR Gutie'rrez, NR Pradhan, L Balicas, TE Mallouk, F Lo'pez Urı'as, H Terrones, M Terrones, ACS Nano 7, 5235 (2013)

65. J-G Song, J Park, W Lee, T Choi, H Jung, CW Lee, S-H Hwang, JM Myoung, J-H Jung, S-H Kim, C Lansalot-Matras, H Kim, ACS Nano 7, 11333 (2013)

66. X Wang, H Feng, Y Wu, L Jiao, J. Am. Chem. Soc. 135, 5304 (2013)

67. Y-H Lee, X-Q Zhang, W Zhang, M-T Chang, C-T Lin, K-D Chang, Y-C Yu, JT-W Wang, C-S Chang, L-J Li, T-W Lin, Adv. Mater. 24, 2320 (2012)

68. Y-H Lee, L Yu, H Wang, W Fang, X Ling, Y Shi, C-T Lin, J-K Huang, M-T Chang, C-S Chang, M Dresselhaus, T Palacios, L-J Li, J Kong, Nano Lett. 13, 1852 (2013)

69. C Mai, A Barrette, Y Yu, YG Semenov, KW Kim, L Cao, K Gundogdu, Nano Lett. 14, 202 (2013)

70. S Wu, C Huang, G Aivazian, JS Ross, DH Cobden, X Xu, ACS Nano 7 , 2768 (2013)

71. A Castellanos-Gomez, R Rolda'n, E Cappelluti, M Buscema, F Guinea, HSJ Van Der Zant, GA Steele, Nano Lett. 13, 5361 (2013)

72. S Najmaei, Z Liu, W Zhou, X Zou, G Shi, S Lei, BI Yakobson, J-C Idrobo, PM Ajayan, J Lou, Nat. Mater. 12, 754 (2013)

73. AM van der Zande, PY Huang, DA Chenet, TC Berkelbach, Y You, G-H Lee, TF Heinz, DR Reichman, DA Muller, JC Hone, Nat. Mater. 12, 554 (2013)

74. Y Yu, C Li, Y Liu, L Su, Y Zhang, L Cao, Sci. Rep. 3, 1866 (2013)

75. H Schmidt, S Wang, L Chu, M Toh, R Kumar, W Zhao, AH Castro Neto, J Martin, S Adam, B O"zyilmaz, G Eda, Nano Lett. 14, 1909 (2014)

76. HR Gutie'rrez, N Perea Lo'pez, AL Elı'as, A Berkdemir, B Wang, R LV, F Lo'pez Urı'as, VH Crespi, H Terrones, M Terrones, Nano Lett. 13, 3447 (2012)

77. Y Zhang, Y Zhang, Q Ji, J Ju, H Yuan, J Shi, T Gao, D Ma, M Liu, Y Chen, X Song, HY Hwang, Y Cui, Z Liu, ACS Nano 7, 8964 (2013)

78. X Wang, Y Gong, G Shi, WL Chow, K Keyshar, G Ye, R Vajtai, J Lou, Z Liu, E Ringe, BK Tay, PM Ajayan, ACS Nano 8, 5125 (2014)

79. J Shaw, H Zhou, Y Chen, N Weiss, Y Liu, Y Huang, X Duan, Nano Res. 7, 1 (2014)

80. J-K Huang, J Pu, C-L Hsu, M-H Chiu, Z-Y Juang, Y-H Chang, W-H Chang, Y Iwasa, T Takenobu, L-J Li, ACS Nano 8, 923 (2013)

81. F Xia, T Mueller, Y-M Lin, P Avouris, Nat. Nanotechnol. 4, 839 (2009)

Submit your manuscript to a SpringerOpen ${ }^{\odot}$ journal and benefit from:

- Convenient online submission

- Rigorous peer review

- Immediate publication on acceptance

- Open access: articles freely available online

- High visibility within the field

- Retaining the copyright to your article

Submit your next manuscript at $>$ springeropen.com 